\title{
Dix ans de recherche sur les prédispositions génétiques au développement de tumeurs
}

L'intérêt récent pour les aspects héréditaires du cancer découle de nos nouvelles capacités à identifier les gènes impliqués dans les maladies humaines. Mais les situations où la survenue d'un cancer apparaît clairement associée à une mutation germinale préexistante sont relativement rares. Aussi, cet intérêt ne serait-il resté que marginal si l'étude des prédispositions monogéniques majeures à développer des tumeurs n'avait débouché sur l'isolement d'un groupe de gènes qui constituent des sites fréquents de survenue de mutations somatiques au début ou au cours de la progression d'un nombre important de cancers. La description de ces gènes complète notre connaissance de la tumorigenèse qui avait débuté par l'identification d'altérations spécifiques des cellules cancéreuses. Il est permis aujourd'hui d'identifier des sujets fortement prédisposés à développer des tumeurs pour lesquelles, bien souvent, il n'existe pas encore de méthodes validées de surveillance. Ces découvertes posent donc des problèmes médicaux qu'il faut maintenant résoudre alors même que, du fait des progrès thérapeutiques dans d'autres branches de

\section{Gilles Thomas}

ADRESSE ET TIRÉS À PART

G. Thomas : directeur de l'U. 434 de l'Inserm. Génétique des tumeurs, Institut Curie, 26 la médecine, le cancer devient progressivement la première cause de mortalité dans nos populations vieillissantes.

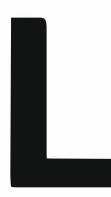

a génétique de la prédisposition au développement des cancers est longtemps restée une curiosité médicale. De rares arbres généalogiques décrivant des concentrations familiales de cancers sont signalés avant le début du $\mathrm{XX}^{\mathrm{e}}$ siècle [1]. Peut-être Watkins a-t-il eu le mérite en 1904 d'être l'un des premiers à s'interroger clairement sur le rôle de l'hérédité dans la survenue de cancers, à un moment où la théorie de Darwin s'imposait définitivement dans les milieux scientifiques [2]. C'est aussi à cette époque et un peu plus tard que Theodore Boveri mentionne l'importance des anomalies caryologiques dans les cellules cancéreuses [3, 4]. $\mathrm{Au}$ cours des décennies suivantes, deux lignes de recherche en cancérologie seront essentiellement poursuivies. L'une explore la base infectieuse du cancer et débouchera sur l'identification de diverses familles de virus oncogènes, particulièrement 
chez les rongeurs, les oiseaux et l'homme. Elle fournira la première voie d'accès aux proto-oncogènes cellulaires. La deuxième ligne de recherche est plus épidémiologique et s'intéresse à la corrélation qui devient progressivement évidente entre les activités cancérogènes et mutagènes de nombreux agents chimiques et physiques. Elle révélera l'importance de l'environnement dans la détermination de l'incidence des cancers [5] et conduira à proposer des méthodes de criblage de substances potentiellement cancérogènes fondées sur la mesure de leur efficacité à induire des mutations dans des souches bactériennes indi- catrices [6]. Elle incitera à spéculer sur l'accroissement exponentiel de la probabilité d'apparition des cancers avec l'âge en supposant qu'il est lié à la nécessité de la survenue, dans la cellule progénitrice, de multiples événements mutationnels indépendants [7-9]. Cette suggestion sera reprise et complétée par P. Nowell en 1975 lorsqu'il expliquera la progression tumorale en proposant que, au cours de l'histoire naturelle d'une tumeur, survient une succession d'expansions clonales des cellules cancéreuses, chaque expansion étant associée à la sélection d'une mutation conférant une plus grande agressivité [10]. Ce processus appa-

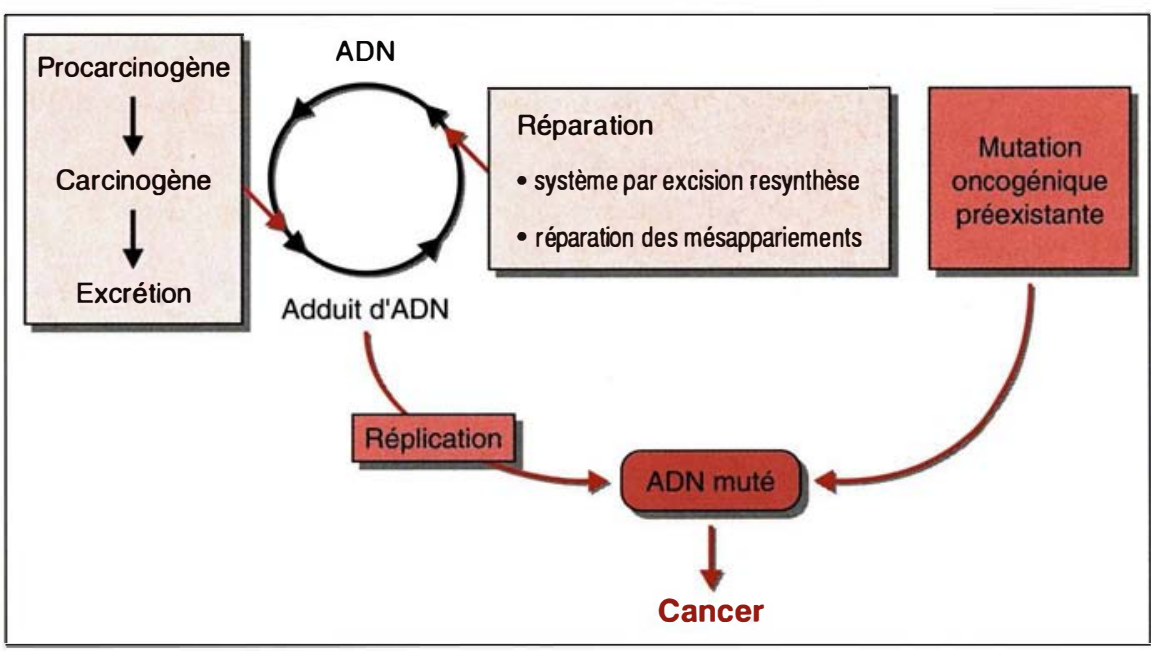

Figure 1. Différents mécanismes modulant la vitesse d'accumulation des mutations. Schématiquement, les prédispositions génétiques à l'apparition de tumeurs sont dues à une augmentation de la vitesse $d^{\prime}$ acquisition des mutations. Quatre groupes principaux de causes peuvent expliquer ce phénomène : (1) L'existence d'une mutation oncogénique héréditaire, c'est-àdire la présence dans la lignée germinale d'une mutation qui, chez les sujets non prédisposés, est habituellement acquise par les cellules néoplasiques au cours de la tumorigenèse. Le plus souvent, ces mutations germinales affectent des gènes suppresseurs de tumeur. A ce jour, la seule exception connue concerne des mutations du gène RET. (2) Des déficits dans les systèmes de maintien de l'intégrité du génome. Les mutations germinales dans les systèmes de réparation par excision resynthèse (xeroderma pigmentosum) et le système de réparation des mésappariements (syndromes HNPCC) sont les plus documentés. (3) Un polymorphisme génétique conduit à une variabilité du métabolisme des xénobiotiques. Cette variabilité individuelle peut moduler le niveau intracellulaire de mutagènes et ainsi conduire à des susceptibilités différentes à développer des cancers. (4) Enfin, la stimulation mitotique qui peut être observée dans certaines maladies inflammatoires ou hormonales à composante héréditaire pourrait favoriser l'accumulation de mutations en entraînant la réplication de l'ADN avant que les réparations nécessaires n'aient pu être effectuées. Ce dernier mécanisme, bien que vraisemblable, reste encore peu documenté. raît d'ailleurs favorisé par l'apparente instabilité génétique observée dans les cellules tumorales qui sera ultérieurement amplement confirmée.

C'est dans cette deuxième voie de recherche que s'inscrit le travail d'Alfred G. Knudson qui, le premier, développera une théorie expliquant la prédisposition génétique au cancer en tenant compte de la nature multiétape du processus mutationnel mis en œuvre dans la tumorigenèse [11, 12]. Par une étude combinant l'épidémiologie génétique et l'analyse des familles présentant des cas multiples de rétinoblastome, il propose que deux événements mutationnels sont nécessaires pour l'apparition de cette tumeur chez un sujet sans antécédent familial. Dans les familles à cas multiples, l'une de ces mutations pourrait être présente dès la conception et donc se retrouver dans toute les cellules de l'organisme. L'apparition de la tumeur nécessiterait une étape mutationnelle de moins. Cette proposition sera affinée par D. Comings dans un article particulièrement clairvoyant où il suggère que ces deux événements pourraient concerner les deux allèles d'un même gène [13]. Ce gène sera plus tard qualifié d'anti-oncogène ou de gène suppresseur de tumeur car l'apparition de la tumeur semble nécessiter l'absence complète de l'expression de ce gène. Nous savons aujourd'hui que cette théorie est avérée pour de nombreuses prédispositions. Nous avons aussi découvert au moins une situation où elle ne s'applique pas. Schématiquement, les prédispositions héréditaires à l'apparition de tumeurs sont dues à des caractéristiques génétiques qui permettent l'accumulation de mutations dans les cellules de l'organisme. Cette accumulation accélérée conduit à atteindre prématurément un nombre critique d'altérations dans une cellule de l'organisme qui donnera naissance à une tumeur. Plusieurs mécanismes peuvent rendre compte de cette prématurité. Les plus importants incluent une instabilité constitutionnelle du génome ou la préexistence d'une lésion oncogénique. Ils peuvent aussi être retrouvés au niveau du contrôle de l'homéostasie dans le système immunitaire, hormonal ou de détoxication. 


\section{Instabilité génomique}

\section{RÉFÉRENCES}

\section{Broca P. Traité des tumeurs. Paris : 1866.}

2. Watkins DJG. A family tree bearing upon the question of the inheritance of cancer. Br Med J 1904 ; i : 190.

3. Boveri T. Uber mehrpolige Mitosen als Mittel zur Analyse des Zellkerns. Wurzburg : Veh Dtsch Zool Ges, 1902.

4. Boveri T. Zur Frage der Entstehung maligner Tumoren. Jena : Fisher, 1914.

5. Doll R, Peto R. The causes of cancer: quantitative estimates of avoidable risks of cancer in the United States today. I Natl Cancer Inst 1981 ; 66 : 1192-308.

6. Ames BN, Durnston WE, Yamasaki E, Lee FD. Carcinogens are mutagens: a simple test system combining liver homogenates for activation and bacteria for detection. Proc Natl Acad Sci USA 1973 ; 70 : 2281-5.

7. Cook PJ, Doll R, Fellingham SA. A mathematical model for the age distribution of cancer in man. Int J Cancer 1969 ; 4:93-112.

8. Dix D. The role of aging in cancer incidence: an epidemiological study. $J$ Gerontol $1989 ; 44: 10-8$.

9. Miller DG. On the nature of susceptibility to cancer. Cancer $1980 ; 46: 1307-18$.

10. Nowell $P$. The clonal evolution of tumor cell populations. Science 1976 ; 194 : 23-38.

11. Knudson AG. Mutation and cancer : statistical study of retinoblastoma. Proc Natl Acad Sci USA 1971 ; 68 : 820-3.

12. Junien $\mathrm{C}$. Les anti-oncogènes. médecine/sciences 1986 ; 2 : 238-45.

13. Commings DE. General theory of carcinogenesis. Proc Natl Acad Sci USA 1973; 70 : n324-8.

14. Sarasin A. La réparation de l'ADN au centre de la biologie de la cellule. médecine/sciences $1994 ; 10$ : 951-2

15. Hoeijmakers JHJ. Human nucleotide excision repair syndrome: molecular clues to unexpected intricacies. Eur J Cancer 1994; $30: 1912-21$.

16. Hoeijmaker JHI, Bootsma D. DNA repair-incision for excision. Nature $1994 ; 371$ : 6545 .

17. Schaffer L, Egly JM. BTF2/TFIIH, un facteur entre transcription et réparation impliqué dans des maladies de la réparation de l'ADN. médecine/sciences $1994 ; 10$ : 973-8.

18. Strathdee CA, Gavish $H$, Shannon I, Buchwald M. Cloning of CDNAs for Fanconi's anemia by functional complementa-
Plusieurs groupes de maladies ont été identifiés comme conférant une sensibilité particulière à des agents génotoxiques (tels que les radiations ionisantes et ultraviolettes et/ou les agents chimiques de pontage des brins d'ADN) et une forte prédisposition à l'apparition de cancers : les plus étudiées sont le xeroderma pigmentosum, l'ataxie télangiectasie, l'anémie de Fanconi, le syndrome de Bloom [14]. Ces maladies se caractérisent par d'autres manif estations (en particulier des troubles hématologiques, neurologiques et immunologiques) qui occupent le devant de la scène, si bien que l'apparition d'un cancer n'en est jamais révélatrice.

La possibilité d'obtenir des lignées cellulaires de ces patients ou des lignées de rongeurs présentant en culture des caractéristiques d'instabilité génétique proches de celles observées chez les sujets affectés a permis, par des expériences tendant à corriger le défaut par des transfections d'ADNc, d'identifier dans plusieurs cas les gènes impliqués. Ainsi, le clonage de gènes, dont les altérations définissent plusieurs groupes de complémentation du xeroderma pigmentosum, a apporté une démonstration définitive que cette maladie est bien due à un déficit dans le système de réparation de l'ADN par excision-resynthèse $[15,16]$. En révélant que les produits de plusieurs de ces gènes sont des sous-unités du facteur de transcription BTF2/TFIIH ce travail a fourni une description moléculaire de la relation bien connue entre la transcription et la réparation [17].

En utilisant une approche analogue, le gène de l'un des quatre groupes de complémentation de l'anémie de Fanconi vient d'être isolé [18]. Malheureusement sa structure ne nous permet pas de prédire la fonction de son produit.

Si trois des quatre groupes de complémentation de l'ataxie télangiectasie ont été localisés précisément sur le chromosome 11 [19], si l'altération responsable du syndrome de Bloom a été localisée sur le chromosome 15 par des techniques élégantes de génétique humaine $[20,21]$, nous ne connaissons pas encore les gènes altérés dans ces affections. Les anomalies chromosomiques observées dans ces syndromes suggèrent, cependant, que le mécanisme du contrôle de la recombinaison pourrait être déréglé.

Toutes ces maladies graves sont à transmission héréditaire récessive. Plusieurs études ont recherché si un état hétérozygote entraînait une prédisposition moins évidente à développer des tumeurs. Elle ne semble pas exister chez les sujets hétérozygotes pour des mutations des gènes du xeroderma pigmentosum, de l'anémie de Fanconi ou du syndrome de Bloom. En revanche, les travaux du groupe de Swift indiquent que les femmes hétérozygotes pour un gène de l'ataxie télangiectasie seraient prédisposées au cancer du sein. Cette étude mérite d'être reproduite car, si ses conclusions étaient avérées, il est estimé que $7 \%$ des femmes atteintes de cancer du sein seraient hétérozygotes pour une telle altération, ce qui ne manquerait pas d'avoir des conséquences sur nos politiques de dépistage [22]

Une autre maladie associée à une anomalie du maintien de l'intégrité du génome vient très récemment d'être identifiée. Elle a été initialement décrite par $\mathrm{H}$. Lynch pour des familles chez lesquelles l'incidence de cancers coliques semblait anormalement élevée. Cet aspect historique explique son nom de HNPCC (pour hereditary non polyposis colon cancer). On sait cependant qu'elle favorise aussi l'apparition de tumeurs de l'endomètre, de l'ovaire, de l'estomac, et des voies biliaires et urinaires, si bien qu'elle a aussi été appelée par les Anglo-Saxons le cancer family syndrome $[23,24]$. Le HNPCC se distingue fondamentalement des affections précédentes sur deux points: l'apparition de tumeurs est son seul mode de révélation; elle se transmet sur un mode dominant. Cette maladie est due à un déficit dans un système de réparation de l'ADN capable de reconnaître des mésappariements. Il est clairement distinct de celui affecté dans le xeroderma pigmentosum. Il a été montré à ce jour que le HNPCC pouvait être provoqué par une mutation sur l'un des gènes d'une famille de quatre gènes localisés sur des segments chromosomiques différents [25-27]. Les cellules des tissus normaux des sujets atteints ne semblent pas souffrir d'in- 
stabilité génétique particulière (encore que ce point mérite sans doute des analyses plus fines). En revanche, les cellules tumorales présentent au niveau de loci particuliers appelés microsatellites (définis par la répétition en tandem de séquences de mono-, di-, tri- ou tétranucléotides) une instabilité caractéristique $\left(\mathrm{m} / \mathrm{s} \quad n^{\circ} 8-9\right.$, vol. 9, p. 990 et $n^{\circ} 5$, vol. 8, p. 500) [28-30]. Dans les tumeurs étudiées, l'unique allèle fonctionnel observé dans les tissus sains a été, soit muté, soit perdu, expliquant ainsi l'instabilité génétique observée [31].

A la différence du xeroderma pigmentosum qui reste exceptionnel, le HNPCC serait relativement fréquent dans la population générale. Il existe, cependant, une incertitude sur sa fréquence exacte. Les estimations de la proportion des porteurs de cancer du côlon qui seraient atteints de HNPCC varient de $1 \%$ (valeur la plus vraisemblable) à plus de $10 \%$.

\section{Inactivation germinale des gènes suppresseurs de tumeur}

La découverte du premier gène suppresseur de tumeur constitue en 1986 un des tout premiers succès d'une nouvelle approche méthodologique de la génétique qui prit en 1992 le nom de clonage positionnel [32]. Il fut en effet possible, il y a une quinzaine d'années, d'entrevoir des stratégies d'isolement de gènes ne nécessitant pas la connaissance préalable des mécanismes physiopathologiques dans lesquels ils sont impliqués. Ces stratégies reposent sur la détermination de la localisation précise des altérations génétiques causales qui est obtenue en appliquant systématiquement et à grande échelle des techniques de génétique moléculaire permettant l'étude de grands fragments d'ADN. Il est notable que la recherche des gènes de prédisposition au cancer a sans doute été déterminante dans le démarrage des grands projets "génomes" qui, dans l'histoire de la recherche biologique, marquera notre décennie.

La découverte du gène $R B$ dont les altérations sont responsables d'une prédisposition à développer des rétinoblastomes et, à un moindre degré, des ostéosarcomes résulte d'une collaboration remarquable entre les cli$m / s n^{\circ} 3$, vol. 11, mars 95 niciens, les cytogénéticiens et les nouveaux spécialistes de la génétique moléculaire $\left(m / s n^{\circ} 1\right.$, vol. 3, p. 55) [33]. Elle a servi d'exemple pour les stratégies de clonage dans un nombre important de désordres du même type. Il fut tout d'abord observé, dans de très rares familles présentant des cas multiples de rétinoblastomes, la présence d'une délétion intercalaire du bras long du chromosome 13 chez les patients atteints. Par ailleurs, les cellules de rétinoblastome se développant éventuellement en absence d'antécédent familial présentaient souvent une altération du chromosome 13 dont un des exemplaires pouvait être totalement perdu. Cependant, des délétions de plus petite taille étaient parfois observées. La survenue de ces pertes de matériel génétique était conforme aux hypothèses émises par Knudson. Les délétions visibles par les techniques de cytogénétique fournissaient une indication sur la localisation du gène responsable, certes précieuse, mais qui restait d'une précision insuffisante pour permettre une identification du gène lui-même puisqu'elles emportaient à chaque fois plusieurs millions de paires de bases.

Il revient à $\mathrm{R}$. White et à son groupe d'avoir appliqué à l'étude des délétions chromosomiques survenant dans les cellules cancéreuses une technique exploitant l'identification réalisée quelques années plus tôt de régions polymorphes du génome. Ces régions dont le nombre croissait rapidement étaient utilisées pour préciser la transmission des gènes au sein de familles afin d'établir des cartes génétiques et de localiser les lésions responsables de maladies génétiques. Imaginant une autre utilisation de ces loci, R. White eut l'idée d'entreprendre une étude comparative des régions polymorphes des ADN extraits du sang et de la tumeur d'un même patient. En identifiant les loci qui présentaient un seul allèle dans l'ADN tumoral alors que l'ADN constitutionnel en présentait deux, il devenait possible de préciser les régions du génome qui avaient été perdues dans les cellules tumorales. Appliquée pour la première fois à l'étude de rétinoblastomes s'étant déclarés chez des sujets dont un des parents avait aussi été atteint, elle devait montrer que le chromosome 13 délé- té ou perdu dans les cellules tumorales provenait du parent non atteint [34]. Cette observation rendait compte des prédictions faites par Knudson et Commings une dizaine d'années auparavant. Plus important encore, la technique démontra l'existence dans les tumeurs de micro-délétions qui n'étaient pas visibles par les techniques cytogénétiques, et dont les limites pouvaient être précisées. Cette approche permit de restreindre la région à explorer à une taille compatible avec une recherche effective de gènes puis, les gènes candidats ayant été identifiés, d'y rechercher des mutations ponctuelles.

Entre 1989 et 1991, trois autres gènes suppresseurs de tumeurs furent isolés. Les autres furent clonés à partir de 1993. Nous connaissons aujourd'hui une dizaine de gènes présentant toutes les caractéristiques prévues par le modèle de Knudson. Les étapes du clonage positionnel sont actuellement considérablement facilitées par la disponibilité de cartes du génome incorporant des données génétiques et physiques (cartes intégrées) et par le développement de techniques permettant la recherche rapide d'exons et de mutations ponctuelles. Aussi est-il vraisemblable que les quelques gènes de prédisposition majeure au développement de cancer qui ne sont pas encore identifiés le seront dans les toutes prochaines années.

\section{Gènes de prédisposition et tumeurs sporadiques}

Il est très généralement observé que des tumeurs se développant dans un contexte sporadique, mais dont le type est connu pour être prédisposé par une mutation germinale d'un gène suppresseur de tumeur présentent une inactivation somatique de ce gène. Cela a été particulièrement bien établi dans le cas du cancer du côlon. Ainsi, des mutations germinales du gène $A P C$, localisé sur le bras long du chromosome 5, confèrent une maladie appelée polypose adénomateuse familiale prédisposant fortement au développement des cancers colorectaux. Cette prédisposition touche en France environ 2000 sujets. Chaque année, grâce aux méthodes de prévention mises en place, moins de 100 cancers colo- 


\section{RÉFÉRENCES}

19. Aurias A. Ataxie-télangiectasie: aspects cliniques, épidémiologiques et génétiques. médecine/sciences 1994 ; 10 : 957-61.

20. German J, Roe AM, Leppert MF, Ellis NA. Bloom syndrome: an analysis of consanguinous families assigns the locus mutated to chromosome band 15q26.1. Proc Natl Acad Sci USA 1994 ; 91 : 6669-73.

21. Woodage $T$, Prasad M, Dixon JW, Selby RE, Romain DR, Columbano-Green LM, Graham D, Kogan PK, Seip JR, Smith A, Trent RJ. Bloom syndrome and maternal uniparental disomy for chromosome 15. Am J Hum Genet 1994 ; 55 : 74-80.

22. Swift M, Morrel D, Massey RB, Chase CL. Incidence of cancer in 161 families affected by ataxia-telangiectasia. $N$ Engl J Med $1991 ; 325$ : 1831-6.

23. Lynch HT, Lynch JF. The Lynch syndromes. Curr Op Oncol 1993 ; 5 : 687-96.

24. Lynch HT, Smyrk TC, Watson P, Lanepa SJ, Lynch JF, Lynch PM, Cavalieri RJ, Boland CR. Genetics, natural history, tumor spectrum, and pathology of hereditary nonpolyposis colorectal cancer: an updated review. Gastroenterology 1993; 104: 1535-49.

25. Leach FS, Nicolaides NC, Papadopoulos $\mathrm{N}$, et al. (35 auteurs). Mutations of a mutS homolog in hereditary nonpolyposis colorectal cancer. Cell 1993 ; 75 : 1215-25.

26. Nicolaides NC, Papadopoulos N, Liu B, et al. (18 auteurs). Mutations of two homologues in hereditary non polyposis colon cancer. Nature 1994 ; 371 : 75-80.

27. Papadopoulos $\mathbf{N}$, Nicolaides $\mathrm{NC}$, Wei YF, et al. (20 auteurs). Mutation of a $M u t L$ homolog in hereditary colon cancer. Science 1994 ; 263 : 1625-8.

28. Aaltonen LA, Peltomaki P, Leach FS, et al. (14 auteurs). Clues to the pathogenesis of familial colorectal cancer. Science 1993 ; $260: 812-6$.

29. Parsons R, Li GM, Longley MJ, Fang WH, Papadopoulos N, Jen J, De la Chapelle A, Kinzler KW, Vogelstein B, Modrid P. Hypermutability and mismatch repair deficiency in RER+ tumor cells. Cell 1993 ; 75 : 1227 36 .

30. Thibodeau SN, Bren G, Schaid D. Microsatellite instability in cancer of the proximal colon. Science $1993 ; 260: 816-9$

31. Hemminki A, Peltomaki P, Mecklin JP Järvinen $H$, Salovaara $R$, Nystrōm-Lahti $M$, De la Chapelle A, Aaltonen LA. Loss of the wild type $M L H 1$ gene is a feature of hereditary nonpolyposis colorectal cancer. Nature Genet 1994 ; 8 : 405-10.

32. Collins F. Positional cloning: let's not call it reverse anymore. Nature Genet 1992 ;
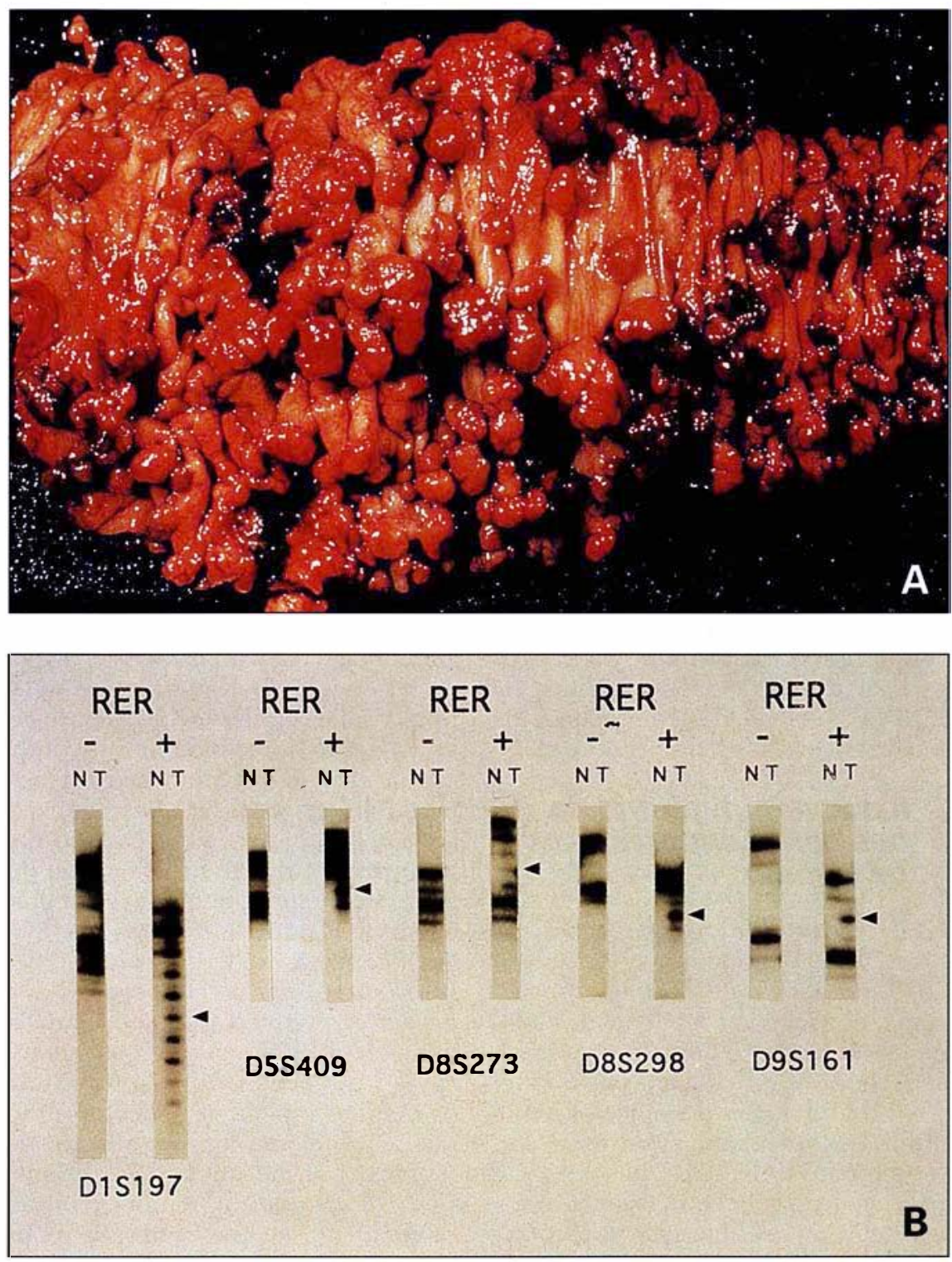

rectaux se développent chez ces patients. En revanche, les cancers colorectaux constituent une maladie très fréquente avec quelque 28000 nouveaux cas par an en France. Dans au moins $60 \%$ des cancers colorectaux, soit dans approximativement $10 \%$ des cancers diagnostiqués dans notre pays, un des allèles du gène $A P C$ est muté et son second allèle est éliminé par une délétion du bras long du chromosome $5\left(\mathrm{~m} / \mathrm{s}, n^{\circ} 9\right.$ vol. 8 , p. 1000 et $n^{\circ} 2$, vol. 10, p. 228) [35, $36]$.

Le gène $B R C A 1$, qui vient récemment d'être isolé $\left(\mathrm{m} / \mathrm{s} n^{\circ} 11\right.$, vol. 10 , p.1172) [37], constitue dans ce contexte peut-être une exception. Il s'agit vraisemblablement d'un gène suppresseur de tumeur : plusieurs altérations germinales détectées dans des familles prédisposées au développement de cancers du sein et/ou de l'ovaire semblent l'inactiver complètement ; les tumeurs du sein qui surviennent chez les femmes de ces familles présentent une perte du bras long du chromosome 17 qui ne porte pas l'altération germinale [38]. Cependant, à ce jour aucune mutation somatique de BRCAl n'a encore été trouvée, suggérant que ce gène ne serait impliqué que dans la forme familiale de cancer du sein associée à sa mutation germinale [39].

Il a été proposé que des gènes de 
Figure 2. Prédispositions génétiques majeures au cancer colorectal. Les prédispositions génétiques majeures au cancer colorectal sont divisées en 2 groupes. Le premier est dû à des altérations du gène APC provoquant l'absence du produit d'un des allèles ou la synthèse d'une protéine tronquée. Dans sa forme caractéristique, la pathologie qui lui est associée, appelée polypose adénomateuse familiale, provoque l'apparition, généralement aux environs de la puberté, de centaines de polypes adénomateux (partie A). Chaque polype est une lésion précancéreuse dont le risque d'évolution vers le carcinome est élevé. Le deuxième groupe est provoqué par une mutation sur un gène dont le produit est essentiel au système de réparation des mésappariements de l'ADN. L'inactivation de ce système par mutation somatique du second allèle se traduit par une instabilité au niveau des loci microsatellites qui n'est observable que sur l'ADN tumoral. Sur la partie B sont comparés les comportements de 5 loci microsatellites pour deux tumeurs coliques. La première indiquée par le signe "-" est stable et possède vraisemblablement un système de réparation des mésappariements fonctionnel. Pour cette tumeur, il n'existe pas de différence entre le typage effectué sur I'ADN provenant de tissu normal (N) et celui provenant de la tumeur (T). En revanche, la tumeur indiquée par un signe "+" présente pour ces cinq loci une instabilité qui est visualisée par l'apparition de bandes supplémentaires dans la partie droite de chaque canal (particulièrement visible sur le locus D1S197). De manière à bien visualiser les différences de comportement entre I'ADN normal et I'ADN tumoral, I'analyse est réalisée dans deux canaux adjacents très rapprochés si bien qu'il n'existe pas sur l'autoradiographie de séparation visible entre les deux. RER: replications errors, signalées par des mutations somatiques ubiquitaires dans les tumeurs coliques comme celles qui se développent dans les familles HNPCC.

prédisposition, particulièrement $R B$ et à un moindre degré $N F 1$, soient l'objet de mutations somatiques fréquentes dans des types tumoraux non prédisposés par leur mutation germinale. Les tumeurs prédisposées par une mutation du gène $R B$ sont essentiellement le rétinoblastome et, à un moindre degré, l'ostéosarcome. Cependant, l'absence d'expression du gène $R B$ est souvent observée dans les lignées cellulaires dérivées de cancer du poumon à petites cellules, plus rarement dans celles dérivées de cancer de la prostate ou du sein. Elle est parfois associée à des mutations identifiées du gène $R B$. La réintroduction de $R B$ dans certaines mais distinct de ce dernier, suggère une autre interprétation à cette observation [45].

Réciproquement, le spectre des tumeurs où un gène suppresseur de tumeur est fréquemment altéré fournit des indications sur l'éventuelle conséquence de sa mutation germinale. C'est sur de telles considérations que $\mathrm{S}$. Friend proposa puis démontra que le gène TP53, dont les mutations somatiques sont retrouvées dans une variété importante de cancers humains, pourrait être altéré dans la lignée germinale de sujets atteints du syndrome de Li et Fraumeni, maladie héréditaire caractérisée par une prédisposition au développement d'un grand nombre de types tumoraux différents [46].

\section{La transmission héréditaire de mutations oncogéniques dominantes}

En dehors des gènes impliqués dans la réparation de l'ADN, la majorité des gènes dont les altérations prédisposent fortement au développement de tumeurs répond aux caractéristiques attendues pour un gène suppresseur de tumeur. Nous ne savions pas jusqu'à l'année dernière si la transmission d'une mutation oncogénique dominante était possible chez l'homme. Une seule affection humaine pouvait se rapprocher de cette situation. Il s'agit du syndrome de McCune-Albright, qui est caractérisé par une hyperactivité endocrine, une polyostose à base de dysplasie fibreuse et des taches café au lait qui peuvent évoquer certains aspects de la neurofibromatose de type 1 . Cette symptomatologie est due à des mutations activatrices d'une protéine $G_{s} \alpha$ (qui appartient au même groupe de protéines que les protéines Ras) $(\mathrm{m} / \mathrm{s}$ $n^{\circ} 2$, vol. $8, p$. 184) [47]. Les altérations sont identiques à des mutations somatiques retrouvées dans des tumeurs de l'hypophyse ou des cancers de la thyroïde. Cette maladie n'est, cependant, pas héréditaire car les mutations ne sont présentes que dans un sous-ensemble des cellules somatiques et ne semblent pas transmissibles à la descendance, suggérant que leur présence dans toutes les cellules de l'organisme est létale. Cette situation chez l'homme contrastait 


\section{RÉFÉRENCES}

33. Friend SH, Bernards R, Rogeli S, Weinberg RA, Rapaport JM, Albert DM, Dryja TP. A human DNA segment with properties of the gene that predisposes to retinoblastoma and osteosarcoma. Nature 1986 ; 323 : 643-6.

34. Cavenee WK, Dryja TP, Phillips RA, Benedict WF, Godbout R, Gallie BL, Murphree AL, Strong LC, White RL. Expression of recessive alleles by chromosomal mechanisms in retinoblastoma. Nature $1983 ; 305$ : 779-84

35. Thomas G, Muleris M, Salmon R. La génétique du cancer colorectal. médecine/ sciences $1988 ; 4: 274-80$.

36. Olschwang S, Laurent-Puig P, Thomas $G$. Expression phénotypique de la polypose adénomateuse familiale. médecine/sciences $1994 ; 10: 454-6$.

37. Miki Y, Swenson J, Shattuck-Eidens D, et al. (44 auteurs). A strong candidate for the breast and ovarian cancer susceptibility gene BRCA1. Science 1995 ; 266 : 66-71.

38. Smith SA, Easton DF, Evans DGR, Ponder BA. Allele losses in the region 17q12-21 in familial breast and ovarian cancer involve the wild type chromosome. Nature Genet $1992 ; 2$ : 128-31.

39. Futreal PA, Liu Q Shattuck-Eidens D, Cochran C, Harshman K, et al. (26 auteurs) BRCAl mutations in primary breast and ovarian carcinomas. Science $1994 ; 266: 120$ 2.

40. Bookstein R, Shew JY, Chen PL, Scully P, Lee WH. Suppression of tumorigenicity of human prostate carcinoma cells by replacing a mutated $R B$ gene. Science 1990 ; 247 : $712-5$.

41. Takahashi R, Hashimoto T, Xu SX, Xu HJ, Matsui T, Miki T, Bigo-Marshall H, Aaronson SA, Benedict WF. The retinoblastoma gene functions as a growth and tumor suppressor in human bladder carcinoma cells. Proc Natl Acad Sci USA 1991 ; 88:525761 .

42. Wang NP, To $\mathrm{H}$, Lee WH, Lee EYHP. Tumor suppressor activity of $R B$ and $p 53$ in human breast carcinoma cells. Oncogene $1993 ; 8: 279-88$.

43. T'Ang A, Varley JM, Chakraborty S, Murphree AL, Fung YKT. Structural rearrangement of the retinoblastoma gene in human breast carcinoma. Science $1988 ; 242$ : $263-6$

44. Bootsma AH, vanEijck C, Schouten KK, Reubi JC, Waser B, Foekens JA, van Pel R, Zwarthoff EC, Lamberts SW, de Klein A. Somatostatin receptor positive primary breast tumors : genetic, patient and tumor charac-

Figure 3. Représentation schématique de la participation aux voies de contrôle de la prolifération cellulaire des gènes de prédisposition au développement de tumeurs. Les produits des gènes pouvant être mutés au niveau germinal sont indiqués en pointillé noir (suppresseurs de tumeur NF1, NF2, APC, Rb, p53, p16MTS) ou en pointillé rouge (uniquement pour Ret qui est le seul produit d'un gène connu pour avoir des formes oncogéniques dominantes héréditaires). Les produits des gènes dont les altérations n'ont été observées chez l'homme qu'au niveau somatique sont indiqués en noir. Les produits des gènes pour lesquels aucune mutation n'a été identifiée chez I'homme, mais dont la transduction par des rétrovirus oncogènes est connue sont indiqués en gris. Seules les mutations fréquentes sont prises en compte. On distingue sur la partie gauche de la figure une région spécialisée de la membrane cellulaire qui participe à l'adhérence des cellules épithéliales. Cette structure semble être un lieu important du contrôle de la prolifération celIulaire. On y trouve des protéines membranaires spécialisées dans l'adhérence et la reconnaissance cellulaires dont certains variants ou mutants ont été associés avec le processus cancéreux (CD44, E-cadhérine). Le produit du proto-oncogène RET, dont la mutation germinale provoque les néoplasies endocriniennes multiples de type 2, possède un domaine structural analogue aux cadhérines et pourrait être localisé à cet endroit. Le produit du gène APC règle peut-être l'interaction entre la E-cadhérine et les caténines. Son interaction avec les microtubules est connue. Le produit du gène NF2 présente une forte analogie avec les protéines ERM qui assurent une jonction entre les filaments $d$ 'actine et la protéine membranaire CD44. Les récepteurs des facteurs de croissance de l'épiderme (EGF-R) et des cellules gliales (GGF-R aussi appelé Neu/ErbB2) ont des structures proches. Les gènes codant pour ces récepteurs sont l'objet de mutations somatiques dans les gliomes (EGF-R) et $d^{\prime}$ amplifications géniques dans les cancers du sein (GGF-R). Leur activité tyrosine kinase active des protéines de la famille Src/Yes, particulièrement abondantes dans les jonctions adhérentes où elles participent au contrôle des interactions moléculaires dans cette région. Sur la partie droite est détaillée la voie de transduction du signal qui passe par les protéines Ras, protéines bien connues pour être l'objet de mutations activatrices dans les tumeurs humaines. Grb2 est un adaptateur à domaines $\mathrm{SH} 2$ et $\mathrm{SH}$, Sos est un facteur d'échange. Cette voie est inhibée par le produit du gène NF1, qui est une GAP (GTPase activating protein). Dans le noyau, la régulation du cycle cellulaire par les cyclines a été en partie clarifiée. Des mutations germinales peuvent affecter des inhibiteurs des kinases dépendantes des cyclines, soit directement $\left(p 16^{\mathrm{MTS}} 1\right)$, soit indirectement à travers le gène p53 (p2 TWAF1). Outre la transactivation du gène p2 jWAF1, le gène $\mathrm{p} 53$ a un rôle dans le contrôle de l'intégrité du génome, du cycle cellulaire et de l'apoptose qui apparaît aujourd'hui relativement complexe. RB intervient directement au niveau de la transcription, notamment par son association au facteur $E 2 F$ qui est un activateur de plusieurs gènes codant pour des oncogènes. Le gène MYC et des membres de sa famille sont activés par translocation chromosomique ou amplification génique (N-MYC) dans plusieurs types tumoraux (tumeurs hématologiques, neuroblastomes). MAPK: mitogen activated protein kinase ; MEK: MAPKK/Extracellular regulated kinase.

avec les observations faites chez la souris où depuis 1984 nous savions, grâce aux travaux du groupe de Leder [48], que la transmission de génération en génération des mutations activatrices d'oncogène est possible sans que cette transmission soit accompagnée de troubles majeurs du développement.

Récemment, des altérations transmissibles du gène RET ont été associées à une prédisposition héréditaire au cancer médullaire de la thyroïde ou à des syndromes plus complexes re- groupés sous le terme de néoplasies endocriniennes multiples de type 2 (MEN2) $\left(m / s n^{\circ} 4\right.$, vol. 10, p. 450). Ce gène est localisé sur le chromosome 10. Cependant, dans les tumeurs se développant chez les sujets prédisposés, il n'y a pas de perte de matériel génétique provenant de ce chromosome. En réalité, la nature des mutations observées sur le gène $R E T$ chez les sujets atteints de MEN2 suggère fortement que l'allèle muté est dominant par rapport à l'allèle normal $[49,50]$. Ainsi, ces mutations trans- 


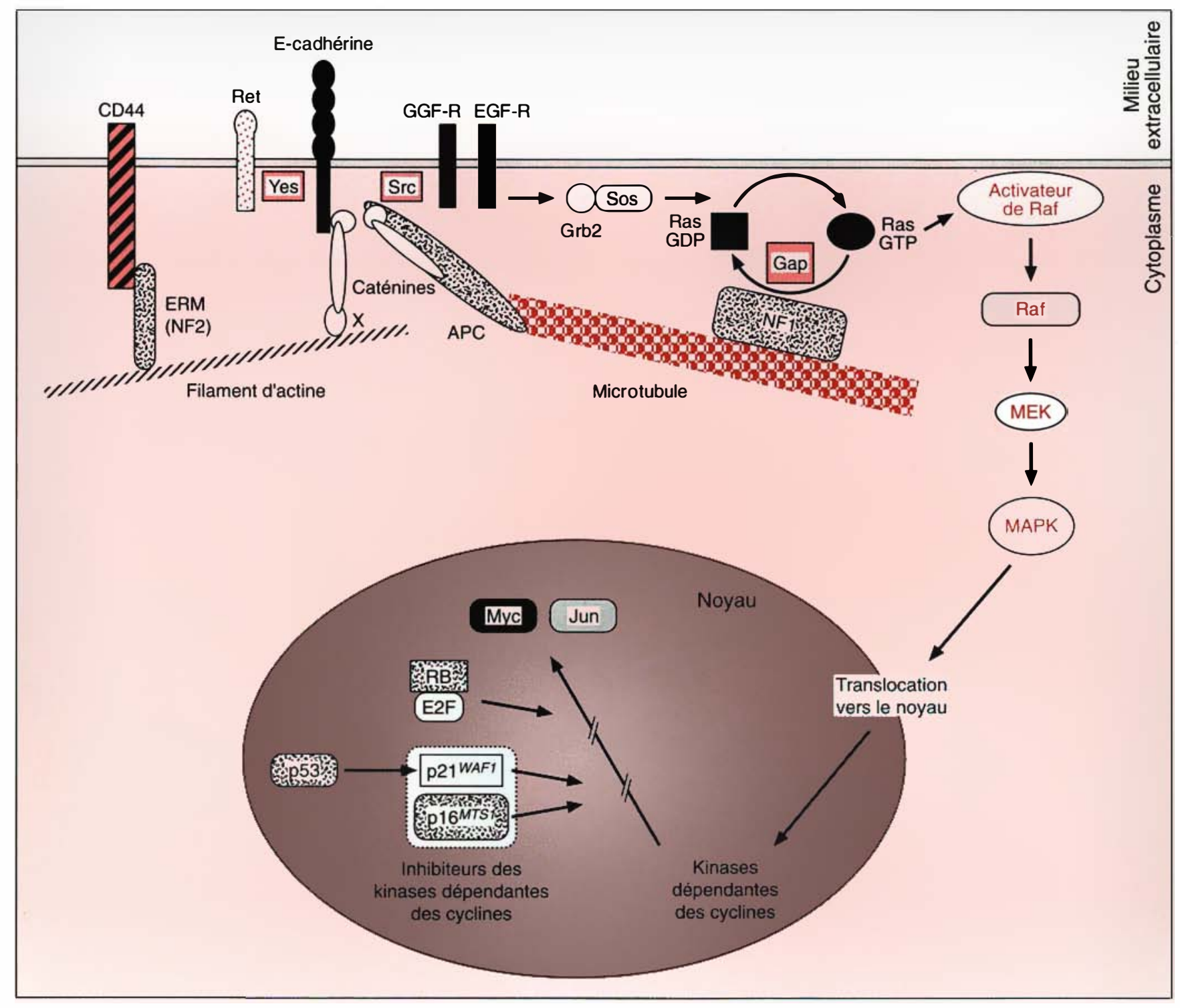

formeraient le proto-oncogène Ret en oncogène, faisant des MEN2 le premier exemple de maladie humaine due à la transmission héréditaire d'un oncogène dominant. Il est à noter que, dans les tumeurs sporadiques de la thyroïde, le gène $R E T$ est très souvent altéré et que les mutations observées conduisent à son activation.

\section{Les gènes cibles de la mutagenèse oncogénique}

L'isolement des gènes de prédispositions majeures au cancer fournit un ensemble de gènes qui vient complé$m / s n^{\circ} 3$, vol. 11 , mars 95 ter notre connaissance des cibles de la mutagenèse oncogénique. Exception faite de $p 53$ dont l'histoire est singulière, et de $D C C$ (deleted in colon cancer) [51] dont le statut n'est pas encore totalement élucidé, tous les gènes suppresseurs de tumeur inactivés par un processus en deux étapes ont été isolés à partir de l'étude des prédispositions génétiques. Ces gènes s'ajoutent à ceux dont les altérations participent à l'initiation tumorale mais qui n'ont jamais été observés mutés au niveau germinal. L'isolement de ce deuxième groupe de gène cibles de la mutagenèse oncogénique a été facilité par leurs implications fréquentes dans des rema- niements chromosomiques visibles par les techniques de cytogénétique (translocations chromosomiques, amplifications géniques) acquis au cours de la tumorigenèse ou par leur capacité, après transfection d'ADN ou d'ADNc dans des cellules en culture, à promouvoir un paramètre du phénotype tumoral. Bien souvent, les gènes identifiés de cette manière avaient été déjà clonés en utilisant des approches expérimentales entièrement différentes, parfois par des groupes ne travaillant pas sur le cancer. Cette redondance dans l'isolement des proto-oncogènes, associée au fait que nous connaissons les gènes de la plupart des prédisposi- 


\section{RÉFÉRENCES}

45. Wooster R, Neuhausen S, Mangion J, et al. (31 auteurs). Localization of a breast cancer susceptibility gene, BRCA2, to chromosome 13q12-13. Science 1994 ; 265 : 208890.

46. Malkin D, Li FP, Strong LC, et al. (11 auteurs). Germ line $p 53$ mutations in a familial syndrome of breast cancer, sarcoma and other neoplasms. Science 1990 ; 250 : 1233-8.

47. Weinstein LS, Shenker A, Geiman V, Merino MJ, Friedman E, Spiegel AM. Activating mutations in the stimulatory $\mathrm{G}$ protein in the McCune-Albright syndrome. $N$ Engl J Med 1991 ; 325 : 1688-95.

48. Stewart TA, Pattengale PK, Leder P. Spontaneous mammary adenocarcinoma in transgenic mice that carry and express transgenic mice that carry and express
$M T V 7 m y c$ fusion gene. Cell $1984 ; 38: 627-$

49. Mulligan M, Kwok JBJ, Healey CS, et al (14 auteurs). Germline mutations of the neoplasia type $2 \mathrm{~A}$. Nature $1993 ; 363$ : 458 60.

50. Santoro M, Carlomagno F, Romano A, et al. Activation of RET as a dominant trans forming gene by germline mutations of MEN2A and MEN2B. Science $1995 ; 267$ : 381-3.

51. Thomas G. Un anti-oncogène membranaire impliqué dans le cancer colorectal. médecine/sciences $1990 ; 6$ : 292-4.

52. Rabbitts TH. Chromosomal translocations in human cancer. Nature $1994 ; 372$ : 143-9.

53. Junien $C$. Un gène candidat pour la tumeur de Wilms. médecine/sciences $1990 ; 6$. 464-9.

54. Ladanyi $M$, Gerald W. Fusion of the EWS and WTI genes in the desmoplastic small round cell tumor. Cancer Res 1994; 54: $2837-40$.

55. Lu SJ, Day NE, Degos L, Lepage V, Wang PC, Chan SH, Simons M, McKnight B, Easton D, Zeng Y, de Thé G. Linkage of a nasopharyngeal carcinoma susceptiblity locus to the HLA region. Nature $1990^{\circ} ; 346^{\circ}$ : $470-1$.

56. Klitz W, Aldrich CL, Fildes N, Horning SI, Begovich AB. Localization of predisposition to Hodgkin disease in the HLA class II tion to Hodgkin disease in the HLA class II
region. Am 9 Hum Genet $1995 ; 54: 497-505$.

57. Apple RJ, Erlich HA, Klitz W, Manos MM, Becker TM, Wheeler CM. HLA DR-DQ associations with cervical carcinoma show papillomavirus-type specificity. Nature Genet $1994 ; 6: 157-62$.

58. Sukumar S, Notario V, Martin-Zance D, Barbacid M. Induction of mammary carcinoma in rats by nitroso-methylurea involves malignant activation of $\mathrm{H}$-ras-1 locus by single point mutations. Nature 1983 ; 306 : tions majeures à développer des tumeurs, suggère que le recensement du répertoire des gènes cibles les plus souvent altérés par la mutagenèse oncogénique est aujourd'hui près d'aboutir.

Il devient possible d'entrevoir les voies de régulations auxquelles participent les gènes suppresseurs de tumeur, les proto-oncogènes et les oncogènes. Si la mutagenèse oncogénique semble avoir comme cible favorite des gènes participant directement à la régulation de la transcription, avec la destruction de facteurs de transcription ou la création de facteurs aberrants engendrés le plus souvent par des translocations chromosomiques [52], il apparaît aussi clairement que les voies de transduction de signaux peuvent aussi être une cible importante. L'identification de mutations activatrices des gènes ras en avait, il y a plus de dix ans, fourni un premier exemple. Les clonages des gènes de prédisposition aux tumeurs en ont ajouté un nombre appréciable (Tableau I).

Les translocations chromosomiques équilibrées conduisant à une activation oncogénique sont retrouvées de manière récurrente dans les tumeurs hématologiques et les sarcomes, alors que les tumeurs d'origine épithéliale sont plus volontiers le site de délétions chromosomiques faisant disparaître une fonction suppresseur de tumeur. Il arrive cependant qu'un même gène joue un rôle de gène suppresseur de tumeur pour une tumeur et soit l'objet d'une conversion oncogénique dans un autre type tumoral. Un exemple particulièrement frappant est le gène WT1 [53] qu'on trouve inactivé dans la tumeur de Wilms alors qu'il est activé par translocation chromosomique dans les tumeurs desmoplastiques in tra-abdominales [54].

\section{Environnement et prédisposition génétique}

Contrairement aux altérations génétiques conduisant à des formes monogéniques de prédispositions majeures au cancer, nous connaissons mal celles qui sont associées à des prédispositions mineures. Nous avons, cependant, quelques indications que la susceptibilité à l'apparition de cancers associés à des facteurs viraux ou environnementaux pourrait être en partie influencée par des facteurs génétiques.

Le locus HLA est l'objet d'une attention particulière. Ainsi, des études de liaison génétique dans la population du sud de la Chine suggèrent qu'un locus de prédisposition lié au locus HLA augmente par 21 le risque de cancer du nasopharynx [55] dont l'association avec le virus d'EpsteinBarr est bien connue. Certains groupements HLA sont plus fréquemment associés à la forme nodulaire sclérosante de la maladie de Hodgkin [56], maladie dans laquelle le virus d'Epstein-Barr semble également impliqué, ou au développement de cancers du col de l'utérus associés au virus papillome de type 16 [57]. Dans ces deux dernières études, les facteurs de risque pour les haplotypes les plus fortement liés restent compris entre quatre et cinq.

L'influence possible des voies de détoxication cellulaire a aussi été examinée. Au cours de la vie, les cellules de l'organisme sont exposées à une grande variété d'agents. Ceux-ci peuvent être d'origine endogène comme, par exemple, les radicaux engendrés par le métabolisme de l'oxygène. Cependant, les agents exogènes jouent pour de nombreux sites tumoraux, sans doute, un rôle prépondérant. La notion qu'ils puissent directement influencer la nature de mutations oncogéniques avait été initialement apportée par l'étude des gènes ras dans un système expérimental de carcinogenèse [58]. L'étude du spectre des mutations pouvant affecter le gène $p 53$ a étendu cette conclusion à l'homme [59]. En particulier, elle a permis de démontrer le rôle direct des dimères de pyrimidine induits par l'exposition au rayonnement ultraviolet dans les cancers de la peau $\left(\mathrm{m} / \mathrm{s} n^{\circ} 5\right.$, vol. $10, p$. 600). Elle a aussi révélé la survenue quasi spécifique de mutations sur le codon 249 dans les cancers du foie chez les sujets exposés à l'aflatoxine B1, ou encore la prépondérance des transversions dans les cancers en rapport direct avec la consommation de tabac $\left(\mathrm{m} / \mathrm{s} n^{\circ} 10\right.$, vol. $5, p$. 789). Tous les organismes vivants ont développé des systèmes de détoxication qui agissent en général en deux étapes. Une première réaction enzymatique engendre des métabolites intermé- 
Tableau I

PRINCIPALES PRÉDISPOSITIONS MONOGÉNIQUES MAJEURES

AU DÉVELOPPEMENT DES TUMEURS A TRANSMISSION DOMINANTE

\begin{tabular}{|c|c|c|c|c|c|c|}
\hline Prédisposition & $\underset{n^{\circ}}{\text { ONIM* }}$ & $\begin{array}{l}\text { Fréquence } \\
\text { (estimée)' }\end{array}$ & $\begin{array}{l}\text { Sites tumoraux } \\
\text { principaux }\end{array}$ & $\begin{array}{l}\text { Localisation } \\
\text { chromosomique }\end{array}$ & $\begin{array}{l}\text { Gène } \\
\text { concerné }^{2}\end{array}$ & $\begin{array}{l}\text { Propriété de } \\
\text { la protéine }\end{array}$ \\
\hline $\begin{array}{l}\text { HNPCC } \\
\text { (syndrome de Lynch) }\end{array}$ & 114400 & $1 / 500$ & $\begin{array}{c}\text { côlon } \\
\text { endomètre } \\
\text { estomac } \\
\text { voies biliaires } \\
\text { et urinaires }\end{array}$ & $\begin{array}{l}2 p \\
3 p \\
2 q \\
7 p\end{array}$ & $\begin{array}{l}\text { MSH2 } \\
\text { MLH1 } \\
\text { PMS1 } \\
\text { PMS2 }\end{array}$ & $\begin{array}{l}\text { participe au } \\
\text { maintien de } \\
\text { l'intégrité du } \\
\text { génome }\end{array}$ \\
\hline $\begin{array}{l}\text { Cancer du sein } \\
\text { héréditaire }\end{array}$ & 113705 & $1 / 500$ & $\begin{array}{c}\text { sein } \\
\text { ovaire } \\
\text { (prostate, côlon) }\end{array}$ & $\begin{array}{l}17 q \\
13 q \\
-\end{array}$ & $\begin{array}{c}B R C A 1 \\
\text { (BRCA2) } \\
-\end{array}$ & $\begin{array}{l}\text { facteur de } \\
\text { transcription } \\
\text { probable }\end{array}$ \\
\hline $\begin{array}{l}\text { Neurofibromatose } \\
\text { de type } 1 \\
\text { (Recklinghausen) }\end{array}$ & 162200 & $1 / 3500$ & $\begin{array}{l}\text { système nerveux } \\
\text { multiples sites }\end{array}$ & $17 q$ & NF1 & $\begin{array}{l}\text { contrôle des } \\
\text { protéines Ras } \\
\text { (type GAP) }\end{array}$ \\
\hline $\begin{array}{l}\text { Polypose } \\
\text { adénomateuse } \\
\text { (syndrome de Gardner) }\end{array}$ & 175100 & $1 / 10000$ & $\begin{array}{l}\text { côlon et rectum } \\
\text { duodénum }\end{array}$ & $5 q$ & $A P C$ & $\begin{array}{l}\text { interactions } \\
\text { avec le } \\
\text { cytosquelette }\end{array}$ \\
\hline Mélanome héréditaire & 155600 & $1 / 10000$ & peau & $\begin{array}{l}9 p \\
1 p\end{array}$ & $\begin{array}{c}\text { MTS1 } \\
-\end{array}$ & $\begin{array}{l}\text { inhibiteur des } \\
\text { kinases } \\
\text { dépendantes } \\
\text { des cyclines }\end{array}$ \\
\hline $\begin{array}{l}\text { Sclérose tubéreuse } \\
\text { de Bourneville }\end{array}$ & 191100 & $1 / 10000$ & $\begin{array}{l}\text { système nerveux } \\
\text { rein }\end{array}$ & $\begin{array}{c}16 p \\
-\end{array}$ & TSC2 & $\begin{array}{l}\text { homologie } \\
\text { avec GAP3 }\end{array}$ \\
\hline $\begin{array}{l}\text { Syndrome de } \\
\text { Li-Fraumeni }\end{array}$ & 114480 & $1 / 30000$ & multiples sites & $17 p$ & p53 & $\begin{array}{l}\text { facteur de } \\
\text { transcription }\end{array}$ \\
\hline $\begin{array}{l}\text { Neurofibromatose } \\
\text { de type } 2 \text { (neurinome } \\
\text { bilatéral du 8) }\end{array}$ & 101000 & $1 / 35000$ & système nerveux & $22 q$ & NF2 & $\begin{array}{l}\text { interaction } \\
\text { cytosquelette- } \\
\text { membrane }\end{array}$ \\
\hline $\begin{array}{l}\text { Rétinoblastome } \\
\text { héréditaire }\end{array}$ & 180200 & $1 / 40000$ & $\begin{array}{l}\text { rétine, } \\
\text { os }\end{array}$ & $13 q$ & $R B$ & $\begin{array}{l}\text { facteur de } \\
\text { transcription }\end{array}$ \\
\hline $\begin{array}{l}\text { Néoplasies endocrines } \\
\text { multiples de type } 1 \\
\text { MEN1 }\end{array}$ & 131100 & $1 / 40000$ & $\begin{array}{l}\text { pancréas } \\
\text { parathyroïde } \\
\text { surrénale } \\
\text { hypophyse }\end{array}$ & $11 q$ & (MEN1) & \\
\hline $\begin{array}{l}\text { Néoplasies endocrines } \\
\text { multiples de type } 2 \\
\text { MEN2 }\end{array}$ & 171400 & $1 / 40000$ & $\begin{array}{l}\text { thyroïde } \\
\text { parathyroïde } \\
\text { surrénales }\end{array}$ & $10 q$ & RET & $\begin{array}{l}\text { récepteur } \\
\text { tyrosine } \\
\text { kinase }\end{array}$ \\
\hline $\begin{array}{l}\text { Maladie de } \\
\text { von Hippel-Lindau }\end{array}$ & 193300 & $1 / 40000$ & $\begin{array}{c}\text { rein } \\
\text { système nerveux }\end{array}$ & $3 p$ & $V H L$ & $\begin{array}{c}\text { protéine } \\
\text { probablement } \\
\text { membranaire }\end{array}$ \\
\hline Syndrome de Gorlin & 109400 & $1 / 60000$ & $\begin{array}{c}\text { rein } \\
\text { système nerveux }\end{array}$ & $9 q$ & $(N B C)$ & \\
\hline $\begin{array}{l}\text { Tumeur de Wilms } \\
\text { (WAGR, Denys-Drash) }\end{array}$ & 194070 & $1 / 100000$ & rein & $11 p$ & WT1 & $\begin{array}{l}\text { facteur de } \\
\text { transcription }\end{array}$ \\
\hline
\end{tabular}

Ne sont pas rapportées dans ce tableau les maladies génétiques à transmission récessive dont les manifestations non tumorales occupent le devant du tableau (xeroderma pigmentosum, ataxie-télangiectasie, anémie de Fanconi, syndrome de Bloom) ou des anomalies immunitaires (syndrome de Wiskott-Aldrich), métaboliques (tyrosinose...) ou chromosomiques de grande taille (trisomie 21 ...) connues pour prédisposer aux tumeurs.

1. Ces fréquences estimées sont présentées à titre indicatif. Elles peuvent varier d'un facteur 2 ou plus.

2. Les gènes BRCA2, MEN1 et NBC ne sont pas encore clonés.

* ONIM: Office de nomenclature internationale des maladies.

$\mathrm{m} / \mathrm{s} n^{\circ} 3$, vol. 11, mars 95 


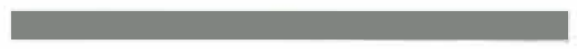

\section{RÉFÉRENCES}

59. Greenblatt MS, Bennett WP, Hollstein $M$, Harris CC. Mutations in the $p 53$ tumor suppressor gene: clue to cancer etiology and molecular pathogenesis. Cancer Res 1994 ; 54 : 4855-78.

60. Vineis D, Bartsch H, Caporaso N, et al. (11 auteurs). Genetically based $\mathrm{N}$-acetyltransferase polymorphism and low-level environmental exposure to carcinogens. Nature 1994 ; 369 : 1546.

61. Nakachi K, Imai K, Kayashi SI, Kawajiri $\mathrm{K}$. Polymorphism of the CYPIA1 and glutathione S-transferase genes associated with susceptibility to lung cancer in relation to cigarettes dose in a Japanese population. Cancer Res 1993 ; 53 : 2994-9.

62. Tefre T, Ryborg D, Haugen A, Nebert DW, Skang V, Brogger A, Borresen AL. Human CYPIAl (cytochrome P450) gene: lack of association between the Mspl restriction fragment length polymorphism and the incidence of lung cancer in a Norweginan population. Pharmacogenetics $1991 ; 1: 20-5$.

63. Caporaso N, Landi MT, Vireis P. Relevance of metabolic polymorphisms to carcinogenesis: evaluation of epidemic evidence. Pharmacogenetics $1991 ; 1: 4-19$.

64. Hirronen A, Husgafvel, Pursiainen $\mathrm{K}$, Antilla S, Karjalainen A, Vaino H. Polymorphism in CYP1A1 and CYP2D6 genes: possible association with susceptibility to lung cancer. Environ Health Persp 1993 ; 101 (suppl. 3) : 109-12

65. Nazarr-Stewart V, Motulsky AG, Eaton DC, et al. The glutathione S-transferase mu polymorphism as a marker for susceptibility to lung carcinoma. Cancer Res 1993 ; 53 : 2313-8.

66. Brockmoller J, Kerb R, Drakoulis N, Nitz $M$, Roost I. Genotype and phenotype of glutathione S-transferases class mu isoenzymes $\mathrm{mu}$ ans psi in lung cancer patients and controls. Cancer Res 1993 ; 53 : 1004-11.

67. Ryberg D, Kure E, Lystad S, Skang V, Stangeland L, Mercy I, Borresen AL, Hangen A. p53 mutations in lung tumours: relationship to putative susceptibility markers for cancer. Cancer Res 1994; $54: 1551-5$.

68. Hayashi S, Watanabe J, Kawajiri K. High susceptibility to lung cancer analysed in terms of combined genotypes of P4501A1 and $\mathrm{mu}$ class glutathione S-transferase genes. Jpn J Cancer $1992 ; 83$ : 866-70.

69. Gibbs JB, Oliff A, Kohl NE. Farnesyltransferase inhibitors: ras research yields a potential cancer therapeutic. Cell $1994 ; 77$ 175-8.

70. Jacks T, Shih TS, Schmitt EM, Bronson RT, Bernards A, Weinberg RA. Tumour predisposition in mice heterozygous for a targeted mutation in NF1. Nature Genet 1994

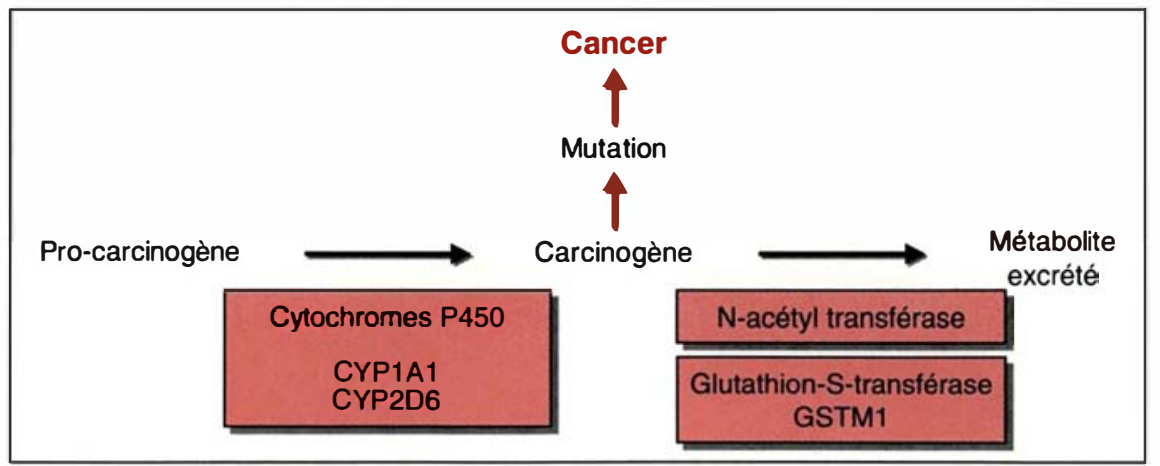

Figure 4. Représentation schématique des deux phases de la détoxication des xénobiotiques. La majorité des pro-carcinogènes sont des molécules hydrophobes dont l'élimination passe par leur transformation en espèces hydrophiles. Ce processus fait en général intervenir deux phases. Dans la première, la substance est activée par une réaction d'oxydo/réduction faisant le plus souvent intervenir une enzyme à cytochrome $P 450$ (CYP). Cette réaction conduit à des métabolites réactifs susceptibles, dans une deuxième phase, de pouvoir se conjuguer à différents groupements hydrophiles (groupement acétyl, glutathion...). Ces métabolites peuvent aussi réagir avec l'ADN, provoquant éventuellement des mutations oncogènes. Différents systèmes enzymatiques interviennent dans ces deux phases; seules sont mentionnées les enzymes qui présentent des polymorphismes dont l'étude suggère qu'ils interviennent dans la susceptibilité individuelle à développer des tumeurs.

diaires possédant un groupement réactif destiné, au cours d'une deuxième étape, à permettre une conjugaison à du glutathion ou à un groupement chimique tel qu'un groupe acétyl. Cette conjugaison conduit à une meilleure solubilité et une excrétion efficace. Cependant, les métabolites intermédiaires présentent l'inconvénient d'être fortement réactifs vis-à-vis de l'ADN. Ainsi, existe-t-il une relation directe chez les fumeurs entre un phénotype d'acétylateur lent et une augmentation d'adduits d'ADN des cellules exfoliées de la vessie [60]. Sans doute parce que les mécanismes de détoxication se trouvent à l'interface entre le milieu extérieur, essentiellement variable, et le milieu intérieur stable, ces systèmes sont inductibles et soumis à des régulations complexes. Leurs gènes présentent aussi de nombreux polymorphismes dans la population humaine. Il est attendu que cette variabilité génétique soit associée à des différences de susceptibilité au cancer dont il faut mesurer l'importance. Les nombreuses études destinées à documenter cette hypothèse ont souvent apporté des conclusions contradictoires. Ces difficultés sont en partie liées au fait que, dans l'approche expérimentale, le génotype est apprécié par une mesure d'activités enzymatiques dont les niveaux d'induction sont difficilement contrôlables. Cette difficulté est actuellement en train d'être surmontée avec l'identification directe des polymorphismes génétiques dont le typage n'est pas soumis à ces aléas. Des données récentes suggèrent que, dans la population japonaise [61] mais peut-être pas dans une population européenne [62], les fumeurs homozygotes pour un allèle du gène CYP1A1*, substituant une isoleucine à une valine et conférant une activité accrue à l'enzyme, ont sept fois plus de risque de développer un cancer $\mathrm{du}$ poumon. Une autre enzyme du même groupe, la CYP2D6, présenterait également des variations génétiques modulant une susceptibilité à ce cancer en activant une nitrosamine du tabac [63]. Des activités accrues combinées de CYP1Al et de CYP2D6 augmenteraient, en synergie, la susceptibilité au cancer du poumon [64].

A l'inverse, les individus dépourvus d'activité GSTM1 (glutathion-S-trans-

* gène codant pour un cytochrome p450, constituant des hydrolases mitochondriales. 
férase mu), due à une délétion homozygote du gène correspondant, seraient plus fréquemment porteurs de cancers. Il apparaît, en particulier, que les sujets ne présentant pas de cancer du côlon, ou présentant un cancer du côlon distal, ou du côlon proximal, ont, respectivement, un génotype nul pour GSTMl dans $42 \%$, $54 \%$ et $70 \%$ des cas [63]. La relation du polymorphisme de cette enzyme avec le cancer du poumon est plus discutée $[65,66]$. Il est intéressant, cependant, de noter que les mutations du gène $p 53$ observées dans les cancers du poumon sont plus fréquemment des transversions si les patients correspondants sont dépourvus de GSTM1 [67]. Enfin, une étude japonaise montre que des fumeurs qui ont, d'une part, le génotype nul pour GSTM1 et, d'autre part, un allèle du gène $C Y P 1 A 1$ à activité élevée, ont un risque relatif de développer un cancer trente fois supérieur à ceux ne présentant aucune de ces deux caractéristiques génétiques [68]. Ce résultat peut se comprendre en suggérant que le génotype CYP1Al contribue à une transformation accrue des procarcinogènes aromatiques présents dans la fumée de cigarette en espèces électrophiles, fortement réactives, dont la détoxication ne peut être réalisée par l'action de la GSTM1 qui est inexistante chez ces sujets.

Les études des prédispositions génétiques multifactorielles au développement de cancers pourraient devenir un enjeu important dans les années à venir. En effet, si la détermination chez un sujet de l'existence d'un facteur mineur de prédisposition ne pourrait à lui seul fournir une information utile à une prise en charge spécifique, la démonstration de la coexistence chez le même individu de plusieurs de ces facteurs pourrait entraîner un risque important méritant des recommandations d'hygiène de vie et une surveillance particulières. Ces facteurs génétiques étant individuellement moins contre-sélectionnés au niveau de la population seraient éventuellement plus largement répandus que les altérations des gènes de prédispositions majeures, et pourraient ainsi contribuer de manière significative à l'incidence des cancers. Cette forme multigénique de prédisposition génétique, bien que majeure au niveau indivi- duel, ne pourrait être repérée par le simple examen des arbres généalogiques.

\section{Le conseil génétique en oncologie génétique}

Le développement de la génétique médicale, et l'affinement des méthodes de la génétique moléculaire fournissent des instruments qui sont pressentis comme devant occuper une place, peut-être importante, dans une meilleure politique de prévention et de dépistage des cancers. Dès maintenant, la possibilité d'évaluer précisément les risques tumoraux des membres de familles atteintes par des prédispositions majeures a l'avantage d'éviter les examens de dépistage inutiles à ceux pour lesquels il a pu être démontré qu'ils n'étaient pas porteurs. Elle permet de cibler la prévention et le dépistage sur les seuls sujets porteurs. Cependant, à de rares exceptions, telles que la polypose adénomateuse familiale, la prise en charge des sujets prédisposés n'a pas obtenu un consensus, faute de données précises sur l'efficacité des stratégies possibles de prévention dans ces situations particulières. Dans la grande majorité des cas, il était pratiquement impossible, avant l'identification des gènes responsables, de réunir un nombre suffisant de sujets de statut génétique connu pour obtenir ces données. Aujourd'hui, l'identification devenant réalisable, les modalités d'utilisation des différentes méthodes de surveillance (coloscopie, mammographie...) doivent faire l'objet d'une évaluation rigoureuse.

Il existe, en particulier, une discussion sur un mode d'intervention radical, la chirurgie préventive. Toute la difficulté d'une décision raisonnée réside dans le fait que l'acte chirurgical qui élimine immédiatement et définitivement le risque tumoral de l'organe enlevé, et l'angoisse qui lui est associée, est indéniablement mutilant et irréversible. Or, à partir de nos connaissances actuelles, il est très difficile de savoir à quelle échéance les progrès dans le dépistage et le traitement des cancers permettront une prise en charge non mutilante et sans risque excessif pour les sujets atteints de prédisposition majeure. Actuellement, dans l'élaboration de la décision thérapeutique, il semble raisonnable de ne prendre en compte que le risque d'apparition de tumeurs au cours des dix prochaines années. Considérer un avenir plus lointain en faisant l'hypothèse que nos méthodes d'intervention n'auront pas progressé est sans doute faire un pari pessimiste.

\section{Vers de nouvelles voies de recherches thérapeutiques?}

La conversion oncogénique d'un proto-oncogène ou l'inactivation des deux allèles d'un anti-oncogène participent au maintien de la cellule tumorale dans un cycle actif de prolifération cellulaire, alors que normalement la cellule aurait dû quitter ce cycle pour entrer en quiescence, en différenciation terminale, en sénescence ou en apoptose. Nous avons aujourd'hui une bonne connaissance descriptive des anomalies génétiques affectant ces gènes. Cette connaissance peut-elle servir de base à la conception de nouvelles voies de recherche thérapeutiques? A travers divers systèmes expérimentaux, in vitro et in vivo dans des modèles animaux, il est apparu que la correction d'une seule des multiples altérations génétiques présentes dans une cellule tumorale conduit généralement à faire régresser ou à inhiber entièrement un paramètre critique du phénotype tumoral, tel que la capacité de se diviser en absence de support solide, ou de former des tumeurs sur des souris nude. Ces observations ont stimulé les tentatives de thérapie génique visant à introduire dans les cellules cancéreuses des éléments génétiques spécifiques inhibant l'expression d'oncogènes ou restaurant l'expression de gènes suppresseurs de tumeur. Bien sûr, pour passer aux applications thérapeutiques, le problème principal reste celui de l'efficacité et de la spécificité du ciblage du gène correcteur dans les cellules tumorales. Deux remarques sont cependant encourageantes: d'une part, l'observation que, au moins dans certaines situations, l'introduction de gènes correcteurs peut avoir un effet majeur sur les cellules tumorales alors qu'elle laisse pratiquement inaltérées les cellules normales ; d'autre part, comme 
le suggèrent les expériences avec les gènes suicides, il est possible que la correction d'une petite proportion des cellules tumorales soit suffisante pour obtenir un effet thérapeutique ( $m / s n^{\circ} 7$, vol. 8, p. 728).

Une voie de recherche thérapeutique alternative consiste à acquérir une connaissance approfondie des mécanismes mis en ouvre par la tumorigenèse, à en disséquer chaque étape pour identifier les molécules impliquées et les interactions qu'elles contractent. L'espoir est d'arriver à trouver une étape qui pourrait faire l'objet d'un contrôle pharmacologique. Actuellement, à titre d'exemple, il apparaît envisageable de tenter d'inhiber, par des méthodes pharmacologiques, une voie de transduction activée par une mutation sur un gène ras ou par l'absence de neurofibromine produit du gène $N F 1$ [69].

De manière à pouvoir tester ces différentes approches thérapeutiques, nous devons à l'avenir développer des modèles animaux qui reproduisent fidèlement les caractéristiques des tumeurs humaines. Les modèles traditionnels de cancérogenèse expérimentale, s'ils ont par le passé apporté des informations importantes, sont limités aujourd'hui par le fait que les tumeurs expérimentales ont souvent des altérations génétiques qui diffèrent de celles observées dans les tumeurs humaines équivalentes. Avec les possibilités actuelles de la transgénèse, il devient possible de reproduire chez l'animal les étapes de la tumorigenèse humaine. Dans cette approche, l'inactivation des homologues des gènes humains de prédisposition aux tumeurs par recombinaison homologue apparaît comme une voie très prometteuse. Ainsi, des modèles murins, correspondant à certaines prédispositions génétiques humaines au développement de tumeurs, ont été récemment obtenus. Dans ces modèles, l'inactivation du second allèle a pu être documentée, suggérant que la tumorigenèse est bien associée à des mécanismes génétiques proches de ceux qui surviennent chez l'homme ([70] et nombreux résultats non publiés). A l'avenir, ces modèles permettront aussi de mieux documenter les rôles respectifs de l'inné et de l'acquis dans l'apparition des tumeurs

\section{Summary}

Ten years of research on the genetic susceptibility to the development of tumors

Our new ability to identify genes involved in human genetic diseases has raised a remarkable interest in the hereditary aspects of cancer. However, situations in which the occurence of cancer can be unambiguously associated with a preexisting germ line mutation are rare. Thus this interest would have deflated rapidly if the study of the major cancer susceptibility diseases had not led to the identification of a group of genes that are the frequent sites of somatic mutations in the initiation or progression of a large proportion of cancers. The description of these genes completes our knowledge of the mechanism of tumorigenesis that had been initiated by the characterization of a different group of genectic alterations which are exclusively observed in cancer cells. Together these findings constitute the conceptual basis on which the major part of basic research on cancer develops. Presently, identification of genetic markers of cancer susceptibility goes beyond the limits of the laboratory. It enables the identification of individuals at high risk of developing cancer for whorn there is an urgent need to determine the optimal follow up. The importance of these findings is underscored by the fact that in developed countries cancer is rapidly becoming the major cause of mortality as a result of the increase of life expectancy.

\section{Remerciements}

Je souhaite remercier particulièrement $A$. Aurias, C. Bonaiti, S. Olschwang et D. StoppaLyonnet pour l'aide apportée à la préparation de ce manuscrit. 